Article

\title{
Energy Saving in Water Distribution Network through Pump as Turbine Generators: Economic and Environmental Analysis
}

\author{
Mauro De Marchis, Barbara Milici, Roberto Volpe and Antonio Messineo * \\ Faculty of Engineering and Architecture, University of Enna Kore, Cittadella Universitaria, Enna 94100, Italy; \\ mauro.demarchis@unikore.it (M.D.M.); barbara.milici@unikore.it (B.M.); roberto.volpe@unikore.it (R.V.) \\ * Correspondence: antonio.messineo@unikore.it; Tel.: +39-935-536-448 \\ Academic Editor: Hua Li \\ Received: 13 July 2016; Accepted: 19 October 2016; Published: 26 October 2016
}

\begin{abstract}
Complex systems of water distribution networks (WDS) are used to supply water to users. WDSs are systems where a lot of distributed energy is available. Historically, this energy is artificially dissipated by pressure reduction valves (PRVs), thanks to which water utilities manage the pressure level in selected nodes of the network. The present study explores the use of economic hydraulic machines, pumps as turbines (PATs) to produce energy in a small network located in a town close to Palermo (Italy). The main idea is to avoid dissipation in favor of renewable energy production. The proposed study is applied to a WDN typical of the Mediterranean countries, where the users, to collect water during the period of water scarcity conditions, install private tanks. The presence of private tanks deeply modifies the network from its designed condition. In the proposed analysis, the economic benefit of PATs application in water distribution networks has been investigated, accounting for the presence of users' private tanks. The analysis, carried out by mean of a mathematical model able to dynamically simulate the water distribution network with PATs, shows the advantage of their installation in terms of renewable energy recovery, even though the energy production of PATs is strictly conditioned by their installation position.
\end{abstract}

Keywords: energy recovery; pump as turbine (PAT); renewable energy; water distribution network; greenhouse gas (GHG)

\section{Introduction}

Water and energy savings are major concerns in the management of water distribution networks (WDNs). Nowadays, energy for pumping represents the main cost for water companies which have to guarantee the delivery of enough water with good quality to population: indeed, water losses reflect directly in energy wasting. This strong connection between water and energy consumption gives rise to programs, such as Watergy [1], aimed at investigating technical and managerial strategies to realize energy, water, and monetary savings through cost-effective actions.

A large effort has been made in recent years to investigate management strategies aimed at reducing net energy consumption in WDNs. Traditional solutions focus on pump and pipe selection, leak detection and repair, and pressure control policies. In fact, water and energy thrifts in WDNs are strictly linked to pipeline water leakages (pipe breaking, joint failures, etc.), which are related to water pressure.

Traditionally, in existing WDNs, head reduction has been achieved by inserting pressure reducing valves (PRVs) [2-5] that are able to maintain pressure variation in a controlled manner. Recently, there has been growing interest in recovering energy instead of dissipating excess pressure through valves, analyzing the potential of turbines to replace PRV or break-pressure tanks, achieving two main 
objectives: leakage reduction and renewable energy production. Unfortunately, the hydraulic turbine installation is a suitable solution just where the available hydraulic power is fairly constant. In fact, where flow rates and heads are highly variable, turbines are not cost effective, so other strategies have been investigated in recent years.

One of the easiest technological solutions, which can combine efficiency and economical convenience, is the use of a centrifugal pump in reverse mode, with the engine acting as a generator [6,7]. Pumps as turbines (PATs) require low cost hydraulic and electric equipment and are often claimed as the cheapest and most sustainable solutions [8] for energy production [9-11], especially for small size power plants $(<40-50 \mathrm{~kW})$. In fact, pumps have various advantages compared to turbines, which are linked to ease of availability, proven technology, low initial installation and maintenance cost, availability for a wide range of heads, and flows [12,13].

The concept of electricity generation through reverse running centrifugal pumps is not new but dates back to around 80 years ago. Recently, Jain and Patel [12] analyzed the historical development and state-of-the-art of PATs, which found application in micro-hydropower plants as well as in water supply piping systems.

Pumps, which work in reverse mode, do not show the same performance when acting in direct (pump) and reverse (turbine) modes, and pumps manufacturers usually provide the characteristic curves only for the direct mode. The evaluation of the characteristic curves of PATs has been one of the most important limitations of their applicability [14-18]. There have been many performance prediction techniques of the turbine operation of pumps based on best efficiency or on specific speed, which have tried to establish a correlation enabling the passage from the direct to the reverse characteristics (see $[13,16]$ for a review). Derakhshan and Nourbakhsh [16] tested several centrifugal pumps and used experimental data to derive some relations to predict the best efficiency point characteristic of PAT. More recently, Nautiyal et al. [8] experimentally tested a centrifugal pump of specific speed to relate pump and turbine mode characteristics. They also used experimental data from other researchers to develop relations to obtain PATs efficiency from pump mode characteristics. These relations gave lower deviation from experimental results respect to those obtained by previous researchers [15-17].

A large number of theoretical and experimental studies have been done for prediction of PAT performance, but there is still a need to explore this area more deeply since the results predicted by these methods are not reliable for pumps with different specific speeds and capacities.

Most recent attempts to predict performance of PATs have been made using computational fluid dynamics (CFD). Unfortunately, in many cases, large deviations in results have been found between experimental and CFD results $[13,15,18,19]$.

Recently, PATs have been investigated in terms of economic benefits. Arriaga [20] carried out the cost analysis for a pico-hydro plant development in the Lao People's Democratic Republic, showing that the implementation of PAT reduces the cost $53 \%$ as compared with cross-flow turbine. Carravetta et al. [19] proposed a method which allows PAT's geometry selection for a given flow-head distribution pattern to find the solution which maximizes the produced energy in WDNs. Carravetta et al. [21] extended the methodology to investigate on best economic efficiency between a hydraulic regulation via a series-parallel hydraulic circuit or an electrical regulation via inverter, finding that hydraulic regulation is more flexible and efficient than electrical regulation. Recently, Puleo et al. [22] investigated energy recovery by PATs in WDNs characterized by the presence of private tanks and intermittent service, highlighting that further analysis was needed for these operating conditions to investigate the possibility and the efficiency of the PATs to recover energy from the WDN. Fontana et al. [23] analyzed a water distribution network in the city of Naples to assess the potential revenues from energy recovery instead of dissipating excess pressure by replacing some of PRVs with PATs, which turned out to guarantee adequate pressure service.

They found that the optimal location of PRVs to reduce water losses did not maximize energy production. Maximum energy recovery needed a multi-objective approach. Nevertheless, results 
showed attractive profits and capital payback period (CPP), which further justify efforts in the analysis of PAT application in WDNs [21,24].

The aim of the present study is to investigate energy recovery in WDNs by PATs to guarantee adequate pressure service and recovery energy from the fluid instead of classical energy dissipation devices (i.e., PRVs). The analysis has been carried out in terms of energy and capital cost recovery.

CFD analysis has been used to analyze energy recovery in WDNs characterized by the presence of private tanks where users who experience water resources rationing store water resources (see $[25,26])$. Such local water storage volumes are actually very common in those countries (e.g., in the Mediterranean area) where water supply is not reliable and users are brought to collect water to cover their needs when the service in unavailable, due to shortage of supply or intermittent distribution [27]. The local private storage interposed between the user and the network is subject to filling/emptying processes, which modify user's demand profile and pressure distribution in the network, causing modifications on PATs energy production capability in the network. Water heads and discharges are calculated through the method of characteristics (MOC). The model, already presented in De Marchis and Freni [28], was used to simulate PATs in small water distribution network.

The above-mentioned research focused the attention of the effect of PATs in water distribution network without actually taking into account the dynamic variation of the energy produced in light of the WDN users' behavior. The proposed mathematical model is able to modify the regulation point of the PAT with the discharge flowing in the WDN, simulating different scenarios and taking into account the presence of private tanks, if installed. The model can thus be considered a management tool for the water utilities to investigate on the possibility of recover energy in WDNs and to optimize the position of the PATs. With respect to other approaches, the proposed mathematical model which is able to simulate a lot of possible work combinations in WDNs (i.e., steady state condition, intermittent distribution, presence of water tanks, etc.).

The model has been applied to the Misilmeri network, a small town located close to Palermo (Italy), characterized by the presence of several private tanks on the roofs, and the results have been analyzed in terms of a management strategy based on the economic analysis and the payback period, taking into account users' satisfaction in terms of water volume supplied in each node for over $24 \mathrm{~h}$.

\section{Mathematical Model and Numerical Procedure}

The mathematical tool has a main part given by the hydrodynamic model, able to simulate complex water distribution networks [25] and a second module able to reproduce the PAT operation mode [28].

The hydrodynamic model solves the momentum and continuity equations using MOC which transform the system of partial differential equations into ordinary differential equations, known as compatibility equations:

$$
\begin{aligned}
& \frac{\mathrm{d} V}{\mathrm{~d} t}+\frac{g \mathrm{~d} h}{c \mathrm{~d} t}+g J_{s}+\frac{g}{c} V \sin (\theta)=0 \\
& \frac{\mathrm{d} V}{\mathrm{~d} t}-\frac{g \mathrm{~d} h}{c \mathrm{~d} t}+g J_{s}-\frac{g}{c} V \sin (\theta)=0
\end{aligned}
$$

where $t$ is the time, $V$ is the velocity averaged over the pipe cross-section, $h$ is the water head, $g$ is the acceleration due to gravity, $c$ is the celerity of pressure waves and $\theta$ is the slope of the pipeline. In the present analysis, a steady state friction model has been used, thus $J_{s}$, that is the head loss per unit length, has been calculated according to the classical formulation by Darcy-Weisbach. The formula is strictly dependent on the roughness of the pipe, which plays a fundamental role in the discharge estimation $[29,30]$. The model is developed to take into account the unsteady friction model of Vítkovský et al. [31] (details can be found in De Marchis and Freni [28]), even though in the present analysis a steady state friction model was used. 
The compatibility equations are valid along the well known characteristic lines (positive and negative):

$$
\begin{aligned}
& C^{+}: \frac{\mathrm{d} s}{\mathrm{~d} t}=+c \\
& C^{-}: \frac{\mathrm{d} s}{\mathrm{~d} t}=-c
\end{aligned}
$$

Equations (1) and (2) can be solved through the finite difference technique and read:

$$
\begin{aligned}
& h_{j}^{i, n+1}-h_{j m}^{i, n}+\frac{c}{g}\left(V_{j}^{i, n+1}-V_{j m}^{i, n}\right)+\left[c J_{j m}^{i, n}+V_{j m}^{i, n} \sin \theta^{i}\right] \Delta t_{i}=0 \\
& h_{j}^{i, n+1}-h_{j v}^{i, n}-\frac{c}{g}\left(V_{j}^{i, n+1}-V_{j v}^{i, n}\right)-\left[c J_{j v}^{i, n}-V_{j v}^{i, n} \sin \theta^{i}\right] \Delta t_{i}=0
\end{aligned}
$$

where $V_{j}^{i, n+1}$ and $h_{j}^{i, n+1}$ are the velocity and the water head in the $j$ th section (of abscissa $(j-1) L_{i} / N_{i}$ ) of the $i$ th pipe at the time step $t^{n}+\Delta t ; \theta^{i}$ is the slope of the $i$ th pipe; $j_{m}$ and $j_{v}$ are the sections upstream and downstream to the $j$ th section, respectively.

The time step advancement $\Delta t_{i}^{n}$, function of the length and the celerity of the $i$ th pipe, is calculated for each pipe and the minimum value is chosen as the unique time step integration:

$$
\Delta t^{n}=\min _{\mathrm{i}} \Delta t_{i}^{n}=\min _{\mathrm{i}}\left(L_{i}^{n} /\left(N_{i} c_{i}\right)\right)
$$

The flow rate into users' tanks is calculated as:

$$
Q_{j, u p}=C_{v} \cdot a \cdot \sqrt{2 g\left(h_{j}^{i}-h_{j, t a n k}\right)}
$$

where $Q_{j, u p}$ is the discharge at the $j$ th node entering the tank connected to the node, $C_{v}$ is the non-dimensional float valve emitter coefficient, $a$ is the valve effective discharge area, $g$ is the gravity acceleration, $h_{j}^{i}$ is the water head at the $j$ th node, and $h_{j, t a n k}^{i}$ is the height of the private tank. Further details on the numerical model can be found in De Marchis et al. [25,27].

The accuracy of the hydrodynamic model was verified comparing numerical results to measured water heads in a real WDN of the city of Palermo (Italy). The comparison, shown in De Marchis et al. [32], clearly showed the ability of the numerical model to predict the variation of the pressure in $24 \mathrm{~h}$. The qualitative agreement between measured and simulated pressure profiles were confirmed by the quantitative analysis carried out calculating the root square mean error (RSME), obtaining an accuracy of the model of about $5 \%$ of the average measured pressure.

To investigate on the energy recovery in water distribution networks, the hydrodynamic model was developed to simulate PAT behavior. Even though several analyses have been carried out to derive the characteristic curve of a water turbine [33], here the characteristic curves achieved by Derakhshan and Nourbakhsh $[15,16]$, through experimental analysis, have been used:

$$
\begin{gathered}
\frac{H_{t}}{H_{t b}}=1.0283 \cdot\left(\frac{Q_{t}}{Q_{t b}}\right)^{2}-0.5468 \cdot\left(\frac{Q_{t}}{Q_{t b}}\right)+0.5314 \\
\frac{P_{t}}{P_{t b}}=-0.3092 \cdot\left(\frac{Q_{t}}{Q_{t b}}\right)^{3}+2.1472 \cdot\left(\frac{Q_{t}}{Q_{t b}}\right)^{2}-0.8865 \cdot\left(\frac{Q_{t}}{Q_{t b}}\right)+0.0452
\end{gathered}
$$

where $H(\mathrm{~m}), P(\mathrm{~W})$, and $Q\left(\mathrm{~m}^{3} / \mathrm{s}\right)$ are head, power, and flow rate, respectively. Subscripts $t$ and $b$ are related to turbine and best efficiency point (BEF).

Following, among others, Derakhshan and Nourbakhsh $[15,16]$ PATs parameters are related to the specific rotational speed per second (rps).

In the present case of study, described in the next paragraph, a pipeline system is investigate, thus justifying the use of $1 \mathrm{D}$ model (MOC method and PAT curve module) to predict the energy recovery 
in urban water distributions. The model is developed thus to work even for flow rate or the pressure conditions which are not compatible with the energy production by means of PATs. In these cases the PATs are by-passed and PRVs are simulated, according to the scheme shown in Figure 1, where turbines are coupled to valves [23].

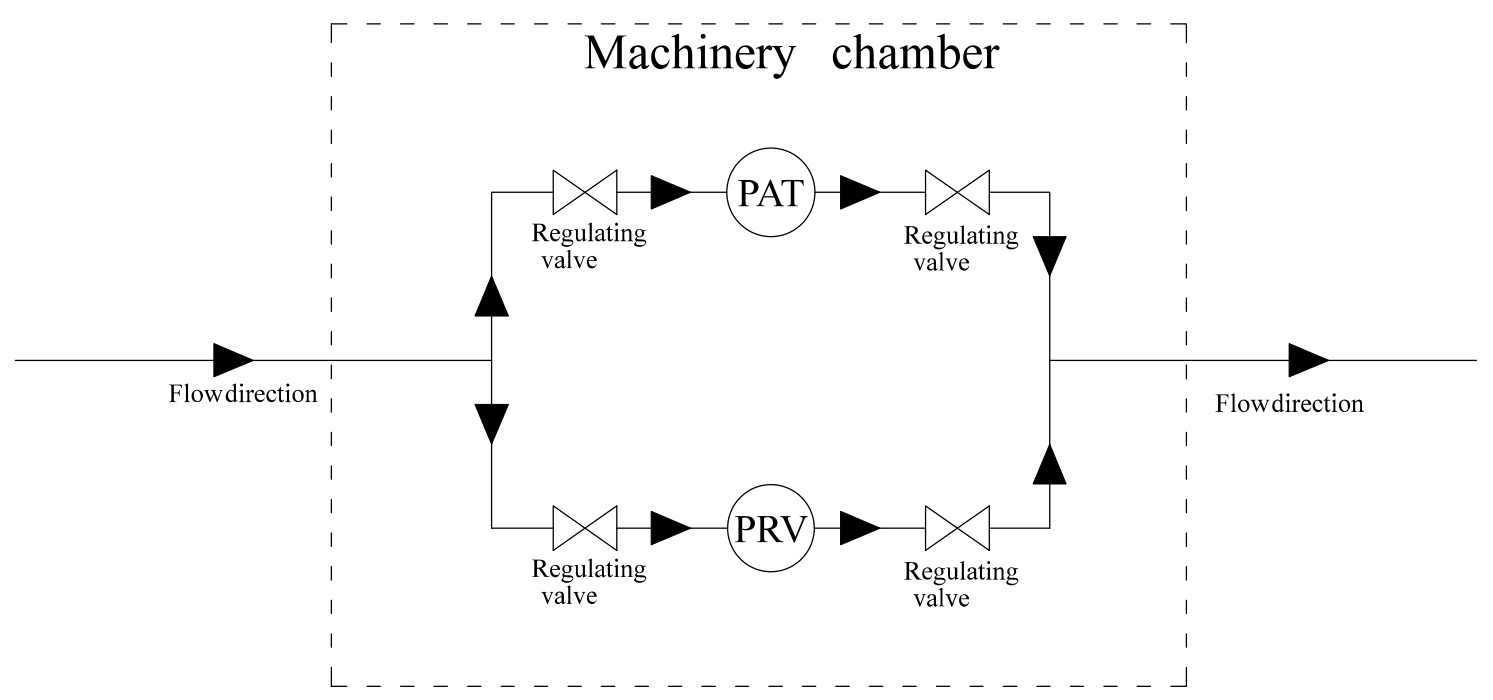

Figure 1. Installation scheme of a coupled system of pumps as turbine (PAT) and pressure reduction valve (PRV).

\section{The Case Study}

The proposed numerical model has been applied to a small town close to Palermo city (Sicily), called Misilmeri, designed to supply about 22,000 inhabitants (11,300 users). The network is made of HDPE and cast iron pipes whose diameters range between 80 and $300 \mathrm{~mm}$. Two main pipelines connected to two water reservoirs feed the network. The first tank, called Castle, is located at $190 \mathrm{~m}$ referred to the mean sea level (MSL) and has a volume of about $500 \mathrm{~m}^{3}$. The second reservoir, known as Cave, is located at $155 \mathrm{~m}$ MSL, having a total water volume of about 1,000 $\mathrm{m}^{3}$.

In Figure 2a, schematic representation of the water network is depicted. As shown, the pipeline system can be subdivided into two sub-network, supplied by the two water tanks. 70 pipes and 57 nodes compose the network under investigation. In Appendix A (Tables A1 and A2), details on the distribution network (diameter, pipe length, water supplied, etc.) are reported. In Appendix A it is also reported the value of the roughness, clearly a key parameter, even though the calibration process (not shown here) pointed out that all the pipes fall into a hydraulically smooth regime, with values of equivalent sand grain roughness $k_{s}$ [34] in the range: $0.001 \mathrm{~mm}<k_{s}<0.005 \mathrm{~mm}$. For this reason, the roughness $k_{s}$ was set equal to the average value $\left(k_{s}=0.003 \mathrm{~mm}\right)$.

The water distribution network configuration is characterized by significant differences between the geodetic quote of water tanks and the users, suggesting high levels of energy recovery. The network orography thus seems to have an excellent configuration in order to test the application of micro energy recovery, introducing PATs in the network pipes.

One of the main problems in PATs positioning is to find the optimal installation point, thus to achieve both maximum energy production and desired water supply. Due to the small number of users, in the present case the choice of the optimal installation point has been carried out following the hydraulic regulation of the network. In small WDNs, the hydraulic regulation can be easily applied, achieving high performance levels, as demonstrated by De Marchis and Freni [28]. 


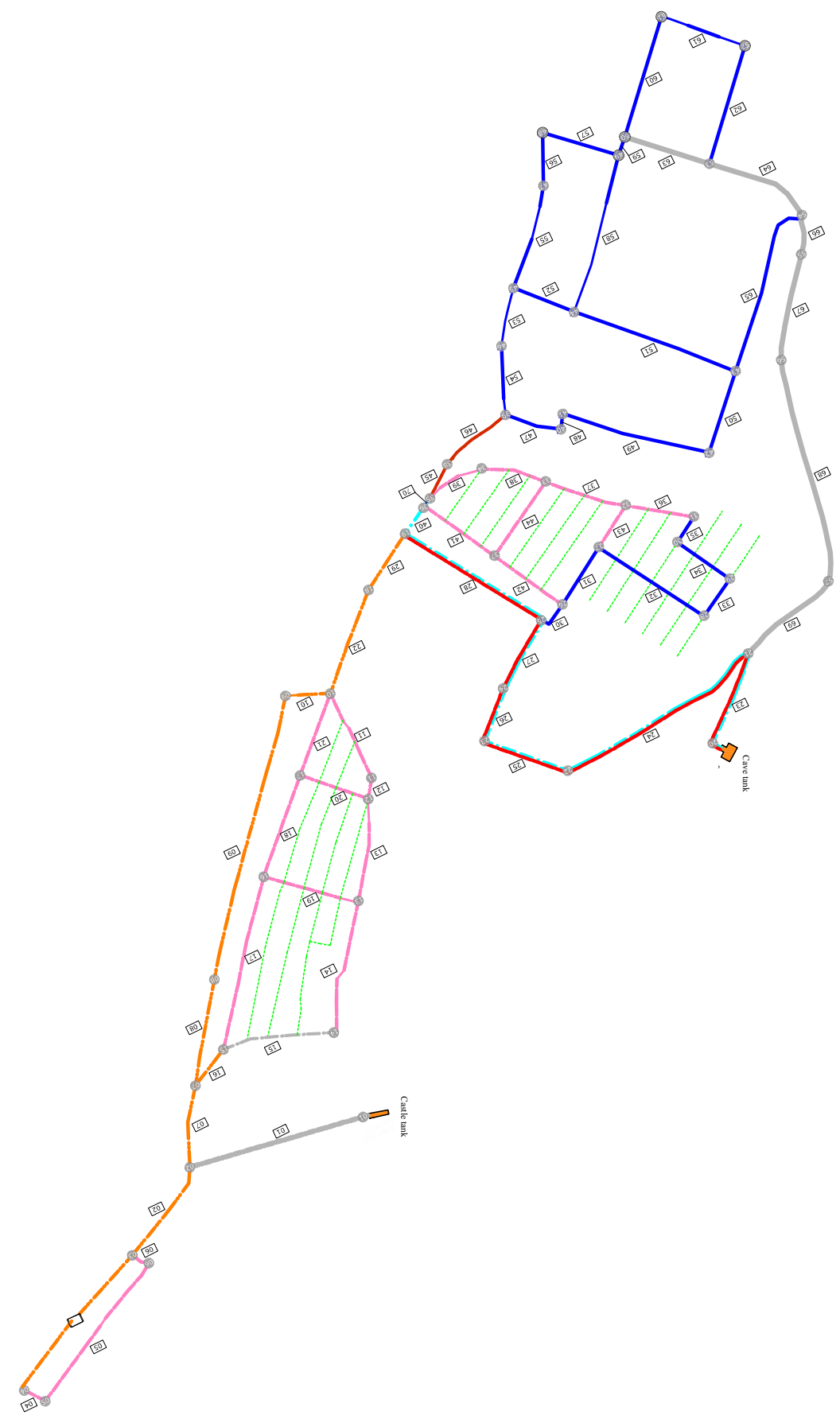

Figure 2. Case study network in Misilmeri (PA). Boxe: pipe number; circle: node number. Colors are representative of pipe diameters. Red line: DN $80 \mathrm{~mm}$; blue line: DN $100 \mathrm{~mm}$; dot dashed gray line: DN 110 mm; dot-dot dashed orange line: DN 150 mm; dashed sky-blue line: DN 200 mm; bold gray line: DN $300 \mathrm{~mm}$.

Taking advantage of the high variation of the geodetic elevation between the water tanks and the network, the main objective of the present analysis is to recover energy, in terms of water head, from water distribution networks.

At the moment, in WDNs the energy is dissipated through float valves. As specified in the following, the cost of electrically regulated valves is similar to the cost of a pump working in turbine mode, with the fundamental difference that PATs have the capability of producing small amounts of renewable energy. 
Due to the specific case of study, three different scenarios have been explored. In the first PAT installation scenario (hereafter referred to TC1), the PAT has been installed in the pipe 23 having a DN equal to $200 \mathrm{~mm}$ and connecting the Cave tank with the WDN. The second scenario (hereafter referred to TC2) analyzed the case of a PAT installed in the connection pipe, number 01, between the Castle tank and the network, having a diameter of $300 \mathrm{~mm}$. In Table 1 some details on the BEPs of the scenarios, used in Equations (9) and (10) of the PAT model, are reported.

Table 1. PATs characteristic in turbine mode.

\begin{tabular}{ccccc}
\hline Scenario & $\boldsymbol{D}_{\text {imp }}(\mathbf{m m})$ & $\boldsymbol{Q}_{\text {BEP }}\left(\mathbf{m}^{\mathbf{3}} \mathbf{s}\right)$ & $\boldsymbol{H}_{\text {BEP }}(\mathbf{m})$ & $\boldsymbol{P}_{\text {BEP }}(\mathbf{k W})$ \\
\hline TC1 & 200 & 0.029 & 9.00 & 1600 \\
TC2 & 300 & 0.015 & 15.00 & 3.00 \\
\hline
\end{tabular}

Finally, a third scenario (hereafter referred to TC3) has been simulated considering the simultaneous installation of both the turbines in the main pipes. The analyzed network, as briefly described above, is characterized by the presence of private tanks that strongly modify the network behavior.

The local reservoirs, installed by the users to cover the water scarcity condition periods, can affect the energy production and the efficiency of the PAT installation. In this framework, the proposed dynamic numerical model was applied to investigate on the effect of the user water tanks in the energy recovery from the WDS.

In the following, $\mathrm{N}$ - will indicate scenarios without the private tanks, whereas with the capital letter T- will specify the cases where user's tanks are considered.

In light of the year to year economic analysis, in the present case a single demand condition was simulated (see Appendix A for details), representative of an average condition of water supply. Even though the demand varies throughout a single day as well as throughout the seasons, the aims of the present research is to give indication about the energy recovery during several years, thus the use of an averaged condition of water demand is clearly justified. A daily demand pattern is request for diurnal analysis of energy recovery.

Considering that average demands are used and a year-to-year analysis is performed, the use of complex mathematical model able to simulate the transient condition could be not necessary. Nevertheless, the presence of private tanks could create small changes in water distribution networks. De Marchis et al. [25], in fact, demonstrated that small water hammer events occur in WDN due to the opening and closing of the ball valves supplying the private tanks. Even though, in the proposed analysis a steady state version of the mathematical model is applied, the presence of user's tanks suggest the use of a model, based on the MOC method, able to take into account for these small dynamic phenomenon, interacting with the PAT module.

\section{Economic Analysis}

The results of a generalized model to simulate both WDN behavior and power production are discussed with the aim of analyzing the energy recovery in water network of small towns. The analysis is based on the average daily energy production ( $A D E P(\mathrm{kWh} /$ day)) obtained by integrating PAT power profile along $24 \mathrm{~h}$, through the equation:

$$
A D E P=\frac{1}{3600} \cdot \frac{\gamma}{1000} \int_{T} q \cdot \eta_{t} \cdot H_{t} \cdot \mathrm{d} t
$$

where $T=86,400 \mathrm{~s}, \eta_{t}$ is the PAT efficiency, $\gamma$ is the specific weight of water, $q$ is the flow rate through the supply pipe, and $H_{t}$ is the head drop due to the PAT, calculated according to equations of the characteristic curves achieved by Derakhshan and Nourbakhsh [15] (Equations (9) and (10)). Average yearly energy production (AYEP (kWh/year)) is calculated assuming that ADEP is generated daily, 
also in consideration that the network supplies a mainly resident population and that an averaged demand pattern was considered as input for the hydraulic model. In Table 2 details on the installed power and related costs are reported.

Table 2. Economic feasibility of PATs installation in a WDN: Production and Cost analysis for cases with no tank (N-) and with tank (T-). EGE: energy generation equipment; $C W C$ : civil work cost; and $M C$ : maintain cost.

\begin{tabular}{ccccccc}
\hline Scenario & $\boldsymbol{P}_{\text {inst }}(\mathbf{k W})$ & $\begin{array}{c}\boldsymbol{A D E P} \\
(\mathbf{k W h} / \text { Day })\end{array}$ & $\begin{array}{c}\boldsymbol{A Y E P} \\
\mathbf{( k W h} / \text { Year })\end{array}$ & $\boldsymbol{E G E ( \boldsymbol { \epsilon } )}$ & $\boldsymbol{C W C}(\boldsymbol{\epsilon})$ & $\begin{array}{c}\boldsymbol{M C} \\
(\boldsymbol{\epsilon} / \text { Year })\end{array}$ \\
\hline N-TC1 & 1.60 & 60.00 & $21,900.00$ & 3800.00 & 1100.00 & 750.00 \\
N-TC2 & 3.00 & 40.00 & $14,600.00$ & 7100.00 & 2100.00 & 1400.00 \\
N-TC3 & 4.60 & 95.00 & $34,700.00$ & $10,800.00$ & 3200.00 & 2100.00 \\
T-TC1 & 1.60 & 40.00 & $14,600.00$ & 3800.00 & 1100.00 & 750.00 \\
T-TC2 & 3.00 & 25.00 & 9100.00 & 7100.00 & 2100.00 & 1400.00 \\
T-TC3 & 4.60 & 58.00 & $21,200.00$ & $10,800.00$ & 3200.00 & 2100.00 \\
\hline
\end{tabular}

Future NCF are actualized to present time by applying a discount rate calculated as the weighted average cost of capital (WACC) of a potential investor.

In the present case, the investor would be a public utility company so that WACC is effectively equal to the current bank interest rate. Here, coherently with the period and the Italian National Bank, it is assumed to be $3 \%$.

The Net Present Value (NPV) is defined by the equation:

$$
N P V=\sum_{j=1}^{N} \frac{(N C F)_{j}+I_{0}}{(1+i)^{j}}
$$

where the $(N C F)_{j}$ is the cash flow at year $j$ related to the conditions under analysis, $I_{0}$ is the initial capital investment which is assumed to financially occur all in year 0 , and $i$ is the WACC.

The $P b T$ and $I R R$ are respectively the time $t$ and the interest rate $i$ at which the following equation is true:

$$
N P V=\sum_{j=1}^{N} \frac{(N C F)_{j}+I_{0}}{(1+i)^{j}}=0
$$

whereas the PI is given by Equation (14):

$$
P I=\frac{N P V}{I_{0}}
$$

Finally, the $R O I$ at year $j$, is defined as:

$$
R O I_{j}=\frac{N C F}{I_{0}}
$$

The economic analysis is based on the use of the MOC model to solve the equation of water distribution networks, presented above. We analyzed two different scenarios related to two different operational condition of the network. In the first one, private tanks are simulated in the hydrodynamic model whereas, in the second, the users are directly connected with the WDN and the presence of private reservoirs is neglected.

The values obtained for NPV, PI, IRR, and ROI are reported on Table 3 calculated assuming the unit energy revenue, equal to $0.22 € / \mathrm{kWh}$ (the current feed in tariff). 
Table 3. Economic indicators for investments regarding cases with no tank (N-) and with tank (T-).

\begin{tabular}{ccccccc}
\hline Indicator & $\mathbf{N - T C 1}$ & $\mathbf{N - T C 2}$ & $\mathbf{N - T C 3}$ & T-TC1 & T-TC2 & T-TC3 \\
\hline$I_{0}(€)$ & 4900 & 9200 & 14,000 & 4900 & 9200 & 4600 \\
$N P V(€)$ & 43,900 & 12,700 & 51,800 & 24,700 & -1600 & 25,700 \\
$P I(\%)$ & 896 & 260 & 1058 & 504 & -34 & 526 \\
$P b T($ Years $)$ & 1.24 & 5.50 & 2.70 & 2.07 & $>12$ & 1.91 \\
ROI (\%) & 83.0 & 20.0 & 39.0 & 51.0 & 7.0 & 55.0 \\
$I R R(\%)$ & 83.0 & 18.0 & 39.0 & 50.5 & 0.5 & 55.0 \\
\hline
\end{tabular}

Table 3 reports the principal economic indicators for investments both in case of no-tanks (N-) and with tanks (T-). Results show that in case of No tanks, Scenario 1 offers the best option in terms of $\mathrm{PbT}$ and IRR, whilst, Scenario 3 offers a better option with respect to NPV.

However, among the No-Tank cases, Scenario 1 is to be considered the preferred one due to a favorable combination between low investment and high NPV. Despite the fact that NPV is higher in Scenario 3, the lower investment of Scenario 1, implies a significant lower risk and significant lower yearly working capital (operation and maintenance expenses).

Among the cases with tanks installed, Scenarios 1 and 3 offer largely similar returns both in terms of NPV and other indicators.

Figures 3 and 4 show results in graphic form for both the No tank and Tank cases.

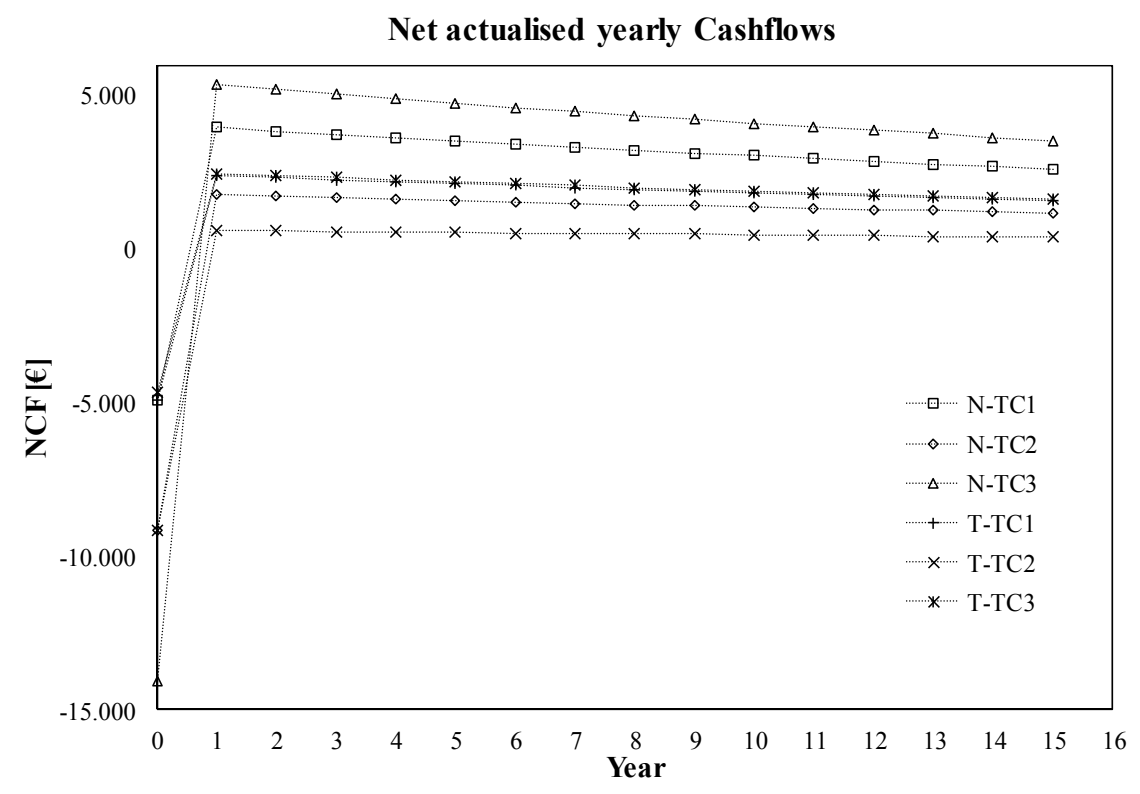

Figure 3. Net actualized cash flows generated in all scenarios.

Figure 4 shows, in particular, how the N-TC3 case becomes more attractive than N-TC1 case only after the seventh year of operation, at which, the cumulated actualized cash flows becomes higher than the N-TC1 case. In addition, Figure 3 shows that the Cases T-TC1 and T-TC3 are very similar in terms of cumulated cash flow, throughout all the period and both considerably more attractive than T-TC2.

The proposed model can be considered an innovative tool, able to guide the water utilities to the optimal positioning of hydraulic machines for the energy saving in WDN. Similarly to the analysis of De Marchis and Freni [28], all the simulations were carried out ensuring the same level of user satisfaction in terms of water supply. 


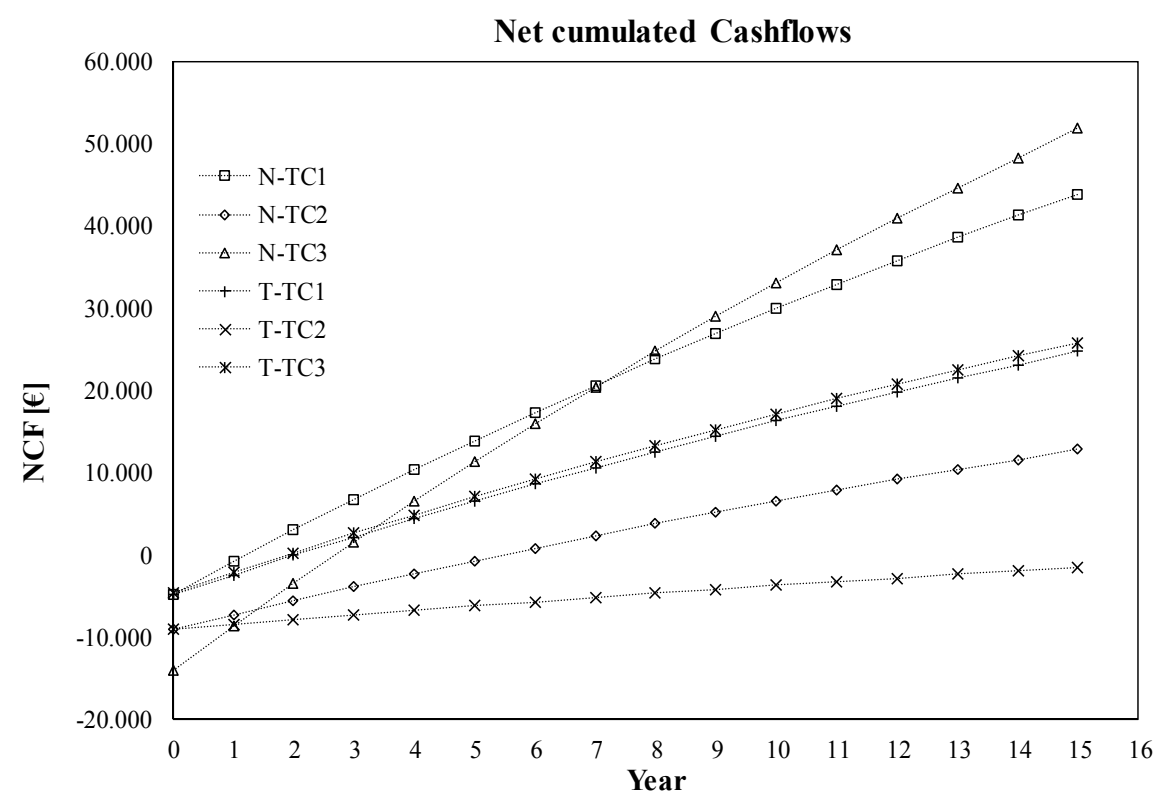

Figure 4. Actualized cumulated cash flows generated by all scenarios.

\section{Environmental Analysis}

In addition to the economic indicators, the installation of PATs implies a significant savings of greenhouse gas (GHG) emissions.

According to the National Energy Authority, the current national energy mix entails a consumption of $0.49 \mathrm{~kg}$ equivalents of $\mathrm{CO}_{2}$ per each $\mathrm{kWh}$ produced [35-38]. The renewable energy produced yearly by PATs in each of the scenarios directly translates into savings of energy which would otherwise be produced by traditional sources, thus in a direct saving of $\mathrm{CO}_{2}$ emission. Figure 5 shows the GHG savings for all the scenarios. The no-tank scenarios induce higher $\mathrm{CO}_{2}$ saving: N-TC1 and N-TC3 are the most beneficial.

\section{GHG saved}

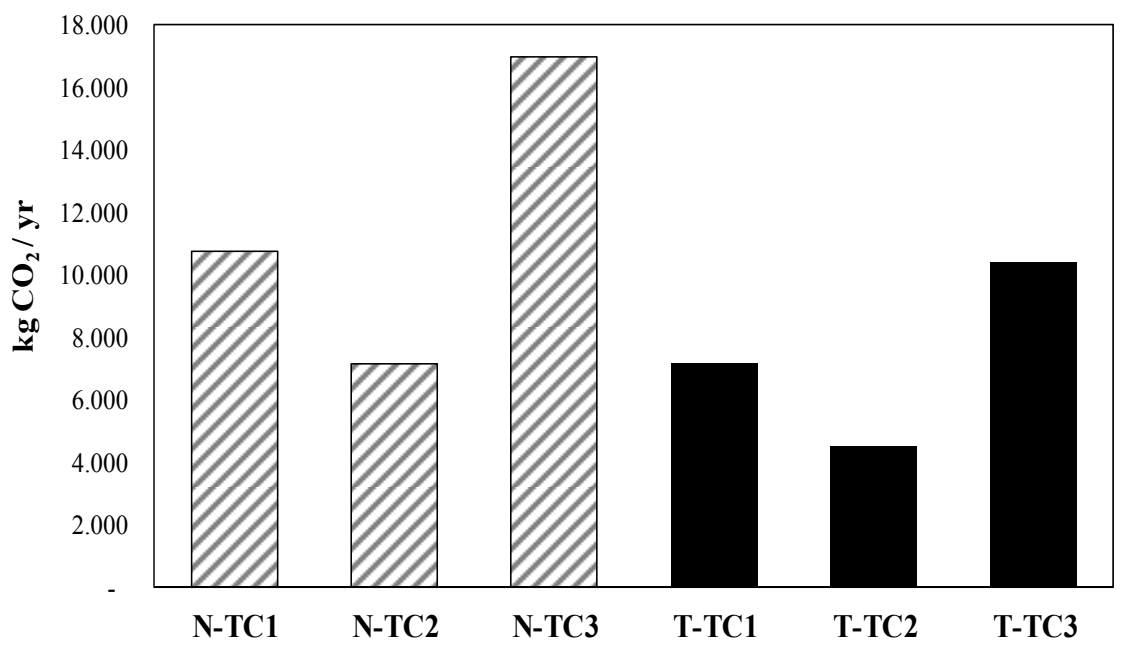

Figure 5. Amount of $\mathrm{CO}_{2}$ saved $(\mathrm{kg})$ yearly in all scenarios analyzed. 
If we now couple the economic results described above with the benefits induced by savings of GHG, we may conclude that N-TC3 is the best scenario. In general terms, should the No-Tank case apply, TC1 is the preferred option from the economic point of view, and TC3 becomes the preferred one if we consider also the environmental benefits. Should instead the Tank case apply, TC3 proves the best option both in economic and environmental terms.

Another environmental consideration worthy of attention is leak reduction. It is known that even though several efforts have been made to reduce hydraulic leakages in WDN, almost all the networks are characterized by water losses. The leakages are strictly connected with the pressure in the networks (see De Marchis et al. [39]) thus the use of PATs, able to produce renewable energy reducing the pressure, represent a double environmental challenge, worthy of extensive future investigations.

\section{Conclusions}

In the proposed study the capability of recover energy in small water distribution network is explored. Basically, a mathematical model was applied to investigate the installation of hydraulic machines known as PATs in small districts of WDNs. The economic and environmental analysis was performed including, in the hydraulic simulation, the presence of private tanks, installed by the users. This way, the results can be considered representative of almost all the networks of the Mediterranean countries, where the practice to use small single reservoirs is well established.

The analysis showed that the use of PATs can be attractive in economic and energy recovery terms, considering the lower capital cost, when compared to traditional turbines. Nevertheless, the PAT installation point affects energy saving. The computed compound Payback Time achieved values of about 2 years or 12 years as a function of the PAT position, clearly showing that, in some cases, the investment is economically unfeasible.

The proposed model can be thus considered a tool, for the water utilities, to maximize the energy saving in WDN. It is useful to specify that all the simulations were carried out ensuring the same level of user satisfaction in terms of water supply.

Overall, the results confirmed that private tanks cause a high decrease of energy production. Considering that, in Mediterranean countries, water utilities have no control on these tanks, to give a more realistic analysis on the performance of energy recovery in WDS, private tanks must be considered.

Finally, the model indicates the possibility of a significant reduction of atmosphere emission of $\mathrm{CO}_{2}$ equivalent greenhouse gases, thus confirming the utility to apply distributed micro energy generators along the several and several WDNs.

Acknowledgments: The authors would like to thank Vincenzo Sucato for the data of the water distribution network of Misilmeri (PA), collected in his degree thesis.

Author Contributions: All the authors contributed to the work in this manuscript. Mauro De Marchis and Barbara Milici mainly contributed to the numerical simulations of the water distribution network model as well as the Pump as Turbine model. Roberto Volpe and Antonio Messineo mainly focused their attention on the economic and environmental analysis. All the authors analyzed the obtained data and contributed to the figure and table generation. All authors revised and approved the manuscript.

Conflicts of Interest: The authors declare no conflict of interest.

\section{Appendix A}

In Appendix A are reported the data of the network used. 
Table A1. Misilmeri network pipe data.

\begin{tabular}{|c|c|c|c|c|c|c|}
\hline Pipe No. & Start Node & End Node & $\begin{array}{c}\text { Diameter } D \\
(\mathrm{~mm})\end{array}$ & $\begin{array}{c}\text { Length } L_{\mathbf{i}} \\
(\mathrm{m})\end{array}$ & $\begin{array}{c}\text { Roughnees } \\
k_{s}(\mathrm{~mm})\end{array}$ & $\begin{array}{c}\text { Discharge } Q \\
(1 / s)\end{array}$ \\
\hline 1 & 1 & 2 & 300 & 200 & 0.003 & 36.33 \\
\hline 2 & 2 & 3 & 150 & 110 & 0.003 & 11.37 \\
\hline 3 & 3 & 4 & 150 & 170 & 0.003 & 7.64 \\
\hline 4 & 4 & 5 & 80 & 32 & 0.003 & 0.97 \\
\hline 5 & 6 & 5 & 80 & 180 & 0.003 & 0.88 \\
\hline 6 & 3 & 6 & 80 & 32 & 0.003 & 2.73 \\
\hline 7 & 7 & 2 & 150 & 90 & 0.003 & 18.96 \\
\hline 8 & 8 & 7 & 150 & 110 & 0.003 & 9.41 \\
\hline 9 & 9 & 8 & 150 & 300 & 0.003 & 8.85 \\
\hline 10 & 10 & 9 & 150 & 44 & 0.003 & 8.29 \\
\hline 11 & 10 & 11 & 80 & 100 & 0.003 & 2.69 \\
\hline 12 & 11 & 12 & 80 & 20 & 0.003 & 2.69 \\
\hline 13 & 12 & 13 & 80 & 110 & 0.003 & 1.51 \\
\hline 14 & 13 & 14 & 80 & 140 & 0.003 & 0.1 \\
\hline 15 & 15 & 14 & 80 & 110 & 0.003 & 4.55 \\
\hline 16 & 15 & 7 & 150 & 44 & 0.003 & 9.03 \\
\hline 17 & 15 & 16 & 80 & 184 & 0.003 & 3.23 \\
\hline 18 & 17 & 16 & 80 & 110 & 0.003 & 0.43 \\
\hline 19 & 13 & 16 & 80 & 110 & 0.003 & 1.79 \\
\hline 20 & 17 & 12 & 80 & 80 & 0.003 & 1.22 \\
\hline 21 & 10 & 17 & 80 & 90 & 0.003 & 2.88 \\
\hline 22 & 18 & 10 & 150 & 120 & 0.003 & 1.93 \\
\hline 23 & 20 & 21 & 200 & 100 & 0.003 & 45.79 \\
\hline 24 & 21 & 22 & 200 & 220 & 0.003 & 36.25 \\
\hline 25 & 22 & 23 & 200 & 100 & 0.003 & 36.25 \\
\hline 26 & 23 & 24 & 200 & 80 & 0.003 & 36.25 \\
\hline 27 & 24 & 25 & 200 & 60 & 0.003 & 36.25 \\
\hline 28 & 25 & 19 & 200 & 170 & 0.003 & 27.98 \\
\hline 29 & 19 & 18 & 150 & 102 & 0.003 & 8.38 \\
\hline 30 & 25 & 26 & 100 & 34 & 0.003 & 8.27 \\
\hline 31 & 27 & 26 & 100 & 58 & 0.003 & 4.84 \\
\hline 32 & 28 & 27 & 100 & 128 & 0.003 & 2.15 \\
\hline 33 & 29 & 28 & 100 & 51 & 0.003 & 0.8 \\
\hline 34 & 29 & 30 & 100 & 64 & 0.003 & 0.11 \\
\hline 35 & 30 & 31 & 100 & 30 & 0.003 & 1.11 \\
\hline 36 & 31 & 32 & 80 & 76 & 0.003 & 1.11 \\
\hline 37 & 32 & 33 & 80 & 85 & 0.003 & 0.27 \\
\hline 38 & 33 & 34 & 80 & 70 & 0.003 & 0.09 \\
\hline 39 & 34 & 35 & 80 & 42 & 0.003 & 0.3 \\
\hline 40 & 36 & 19 & 200 & 38 & 0.003 & 19.6 \\
\hline 41 & 36 & 37 & 80 & 88 & 0.003 & 0.48 \\
\hline 42 & 37 & 26 & 80 & 84 & 0.003 & 2.16 \\
\hline 43 & 32 & 27 & 80 & 51 & 0.003 & 1.29 \\
\hline 44 & 37 & 33 & 80 & 84 & 0.003 & 1.06 \\
\hline 45 & 35 & 38 & 150 & 25 & 0.003 & 17.59 \\
\hline 46 & 38 & 39 & 150 & 82 & 0.003 & 17.59 \\
\hline 47 & 40 & 39 & 100 & 60 & 0.003 & 6.83 \\
\hline 48 & 41 & 40 & 100 & 20 & 0.003 & 4.87 \\
\hline 49 & 42 & 41 & 100 & 154 & 0.003 & 4.87 \\
\hline 50 & 43 & 42 & 100 & 90 & 0.003 & 2.39 \\
\hline 51 & 43 & 44 & 100 & 170 & 0.003 & 1.55 \\
\hline 52 & 45 & 44 & 100 & 74 & 0.003 & 3.27 \\
\hline 53 & 45 & 46 & 100 & 56 & 0.003 & 7.38 \\
\hline 54 & 46 & 39 & 100 & 74 & 0.003 & 7.38 \\
\hline 55 & 45 & 47 & 100 & 112 & 0.003 & 2.81 \\
\hline 56 & 47 & 48 & 100 & 54 & 0.003 & 2.81 \\
\hline 57 & 48 & 49 & 100 & 74 & 0.003 & 1.06 \\
\hline
\end{tabular}


Table A1. Cont.

\begin{tabular}{ccccccc}
\hline Pipe No. & Start Node & End Node & $\begin{array}{c}\text { Diameter } \boldsymbol{D} \\
(\mathbf{m m})\end{array}$ & $\begin{array}{c}\text { Length } \mathbf{L}_{\mathbf{i}} \\
\mathbf{( m )}\end{array}$ & $\begin{array}{c}\text { Roughnees } \\
\boldsymbol{k}_{\mathbf{s}} \mathbf{( \mathbf { m m } )}\end{array}$ & $\begin{array}{c}\text { Discharge } \mathbf{Q} \\
\mathbf{( 1 / \mathbf { s } )}\end{array}$ \\
\hline 58 & 44 & 49 & 100 & 170 & 0.003 & 1.87 \\
59 & 49 & 50 & 100 & 24 & 0.003 & 2.93 \\
60 & 50 & 51 & 100 & 128 & 0.003 & 1.26 \\
61 & 52 & 51 & 100 & 92 & 0.003 & 0.36 \\
62 & 53 & 52 & 100 & 106 & 0.003 & 1.63 \\
63 & 50 & 53 & 100 & 94 & 0.003 & 0.96 \\
64 & 54 & 53 & 100 & 86 & 0.003 & 5.41 \\
65 & 54 & 43 & 100 & 195 & 0.003 & 2.63 \\
66 & 54 & 55 & 110 & 50 & 0.003 & 9.54 \\
67 & 55 & 56 & 110 & 114 & 0.003 & 9.54 \\
68 & 56 & 57 & 110 & 255 & 0.003 & 9.54 \\
69 & 57 & 21 & 110 & 110 & 0.003 & 9.54 \\
70 & 35 & 36 & 200 & 24 & 0.003 & 19.55 \\
\hline
\end{tabular}

Table A2. Misilmeri network node data.

\begin{tabular}{|c|c|c|c|}
\hline Node ID & Node Elevation $z(\mathrm{~m})$ & Water Head $H(\mathrm{~m})$ & Demand $q(1 / s)$ \\
\hline 1 & 190 & 190 & 0 \\
\hline 2 & 141.5 & 189.75 & 1.4 \\
\hline 3 & 143 & 189.23 & 1 \\
\hline 4 & 145.4 & 188.87 & 4.32 \\
\hline 5 & 150.5 & 188.84 & 0.2 \\
\hline 6 & 147 & 188.99 & 0.2 \\
\hline 7 & 137.2 & 188.58 & 0.52 \\
\hline 8 & 135.25 & 188.22 & 0.56 \\
\hline 9 & 126.1 & 187.36 & 0.56 \\
\hline 10 & 128.45 & 187.25 & 4.65 \\
\hline 11 & 134.8 & 186.49 & 0 \\
\hline 12 & 134.9 & 186.34 & 2.4 \\
\hline 13 & 148 & 186.07 & 3.2 \\
\hline 14 & 158.3 & 186.07 & 4.65 \\
\hline 15 & 138.5 & 188.45 & 1.25 \\
\hline 16 & 137 & 186.44 & 1.87 \\
\hline 17 & 132 & 186.47 & 1.23 \\
\hline 18 & 132.5 & 187.27 & 6.45 \\
\hline 19 & 135 & 187.53 & 0 \\
\hline 20 & 195 & 195 & 0 \\
\hline 21 & 186 & 193.35 & 45.79 \\
\hline 22 & 181 & 191.07 & 0 \\
\hline 23 & 154.5 & 190.03 & 0 \\
\hline 24 & 156 & 189.2 & 0 \\
\hline 25 & 156 & 188.58 & 0 \\
\hline 26 & 156 & 187.85 & 1.27 \\
\hline 27 & 154 & 187.42 & 1.39 \\
\hline 28 & 172 & 187.23 & 1.35 \\
\hline 29 & 166 & 187.21 & 0.91 \\
\hline 30 & 158 & 187.22 & 1 \\
\hline 31 & 154 & 187.23 & 0 \\
\hline 32 & 150 & 187.33 & 0.46 \\
\hline 33 & 143.1 & 187.33 & 0.87 \\
\hline 34 & 139.5 & 187.34 & 0.21 \\
\hline 35 & 135 & 187.34 & 1.67 \\
\hline 36 & 135 & 187.41 & 0.53 \\
\hline 37 & 146 & 187.44 & 0.62 \\
\hline 38 & 134 & 187.06 & 0 \\
\hline
\end{tabular}


Table A2. Cont.

\begin{tabular}{cccc}
\hline Node ID & Node Elevation $z(\mathbf{m})$ & Water Head $H(\mathbf{m})$ & Demand $\boldsymbol{q}(\mathbf{l} / \mathbf{s})$ \\
\hline 39 & 131 & 186.14 & 3.38 \\
40 & 136 & 185.25 & 1.96 \\
41 & 136 & 185.1 & 0 \\
42 & 156 & 183.94 & 2.48 \\
43 & 159 & 183.78 & 3.47 \\
44 & 134.5 & 183.65 & 2.95 \\
45 & 129.5 & 183.9 & 1.3 \\
46 & 130 & 184.86 & 0 \\
47 & 132.5 & 183.62 & 0 \\
48 & 133.5 & 183.48 & 1.75 \\
49 & 143 & 183.45 & 0 \\
50 & 143 & 183.39 & 2.63 \\
51 & 142.5 & 183.32 & 1.62 \\
52 & 149 & 183.33 & 1.27 \\
53 & 150 & 183.42 & 2.82 \\
54 & 164 & 184.21 & 1.5 \\
55 & 165 & 185.08 & 0 \\
56 & 168 & 187.05 & 0 \\
57 & 183 & 191.45 & 0 \\
\hline
\end{tabular}

\section{References}

1. Barry, J.A. WATERGY: Energy and Water Efficiency in Municipal Water Supply and Wastewater Treatment Cost-Effective Savings of Water and Energy; The Alliance to Save Energy: Washington, DC, USA, 2007.

2. Tucciarelli, T.; Criminisi, A.; Termini, D. Leak analysis in pipeline systems by means of optimal valve regulation. J. Hydraul. Eng. 1999, 125, 277-285. [CrossRef]

3. Walski, T.; Bezts, W.; Posluzny, E.; Weir, M.; Withman, B. Modeling leakage reduction through pressure control. J. Am. Water Works Assoc. 2006, 98, 147-155.

4. Araujo, L.; Ramos, H.; Coelho, S. Pressure control for leakage minimisation in water distribution systems management. Water Resour. Manag. 2006, 20, 133-149. [CrossRef]

5. Nazif, S.; Karamouz, M.; Tabesh, M.; Moridi, A. Pressure management model for urban water distribution networks. Water Res. Manag. 2010, 24, 437-458. [CrossRef]

6. Chapallaz, J.; Eichenberger, P.; Fischer, G. Manual on Pumps Used as Turbines; Friedr. Vieweg \& Sohn Verlagsgesellschaft mbH: Braunschweig, Germany, 1992.

7. Ramos, H.; Borga, A. Pumps as turbines: An unconventional solution to energy production. Urban Water 1999, 1, 261-263. [CrossRef]

8. Nautiyal, H.; Kumar, V.A.; Yadav, S. Experimental investigation of centrifugal pump working as turbine for small hydropower systems. Energy Sci. Technol. 2011, 1, 79-86.

9. Giugni, M.; Fontana, N.; Portolano, D. Energy saving policy in water distribution networks. In Proceedings of the International Conference on Renewable Energies and Power Quality (ICREPQ'09), Valencia, Spain, 15-17 April 2009.

10. Milici, B.; De Marchis, M. Statistics of inertial particle deviation from fluid particle trajectories in horizontal rough wall turbulent channel flow. Int. J. Heat Fluid Flow 2016, 60, 1-11. [CrossRef]

11. Carravetta, A.; del Giudice, G.; Fecarotta, O.; Ramos, H. PAT design strategy for energy recovery in water distribution networks by electrical regulation. Energies 2013, 6, 411-424. [CrossRef]

12. Jain, S.V.; Patel, R.N. Investigations on pump running in turbine mode: A review of the state-of-the-art. Renew. Sustain. Energy Rev. 2014, 30, 841-868. [CrossRef]

13. Nautiyal, H.; Kumar, V.A. Reverse running pumps analytical, experimental and computational study: A review. Renew. Sustain. Energy Rev. 2010, 14, 2059-2067. [CrossRef]

14. Williams, A.A. Pump as turbines for low cost microhydropower. Renew. Energy 1999, 9, 1227-1234. [CrossRef]

15. Derakhshan, S.; Nourbakhsh, A. Experimental study of characteristic curves of centrifugal pumps working as turbines in different specific speeds. Exp. Therm. Fluid Sci. 2008, 32, 800-807. [CrossRef] 
16. Derakhshan, S.; Nourbakhsh, A. Theoretical, numerical and experimental investigation of centrifugal pumps in reverse operation. Exp. Therm. Fluid Sci. 2008, 32, 1620-1627. [CrossRef]

17. Singh, P.; Nestmann, F. Internal hydraulic analysis of impeller rounding in centrifugal pumps as turbines. Exp. Therm. Fluid Sci. 2011, 35, 121-134. [CrossRef]

18. Yang, S.S.; Derakhshan, S.; Kong, F.Y. Theoretical, numerical and experimental prediction of pump as turbine performance. Renew. Energy 2012, 48, 507-513. [CrossRef]

19. Carravetta, A.; del Giudice, G.; Fecarotta, O.; Ramos, H. Energy production in water distribution networks: A PAT design strategy. Water Resour. Manag. 2012, 26, 3947-3959. [CrossRef]

20. Arriaga, M. Pump as turbine-A pico-hydro alternative in Lao People's Democratic Republic. Renew. Energy 2010, 35, 1109-1115. [CrossRef]

21. Carravetta, A.; Fecarotta, O.; Sinagra, M.; Tucciarelli, T. Cost-benefit analysis for hydropower production in water distribution networks by pump as turbine. J. Water Resour. Plan. Manag. 2014, 140. [CrossRef]

22. Puleo, V.; Fontanazza, C.M.; Notaro, V.; De Marchis, M.; Freni, G.; la Loggia, G. Pumps as turbines (PATs) in water distribution networks affected by intermittent service. J. Hydroinform. 2014, 16, 259-271. [CrossRef]

23. Fontana, N.; Giugni, G.; Portolano, D. Losses reduction and energy production in water-distribution networks. J. Water Resour. Plan. Manag. 2012, 138, 237-244. [CrossRef]

24. Motwani, K.H.; Jain, S.V.; Patel, R.N. Cost analysis of pump as turbine for pico hydropower plants-A case study. Proc. Eng. 2013, 51, 721-726. [CrossRef]

25. De Marchis, M.; Fontanazza, C.M.; Freni, G.; la Loggia, G.; Napoli, E.; Notaro, V. Analysis of the impact of intermittent distribution by modelling the network-filling process. J. Hydroinform. 2011, 13, 358-373. [CrossRef]

26. Freni, G.; De Marchis, M.; Napoli, E. Implementation of pressure reduction valves in a dynamic water network model. J. Hydroinform. 2014, 16, 207-217. [CrossRef]

27. De Marchis, M.; Milici, B.; Freni, G. Pressure-discharge law of local tanks connected to a water distribution network: Experimental and mathematical results. Water 2015, 7, 4701-4723. [CrossRef]

28. De Marchis, M.; Freni, G. Pump as turbine implementation in a dynamic numerical model: Cost analysis for energy recovery in water distribution network. J. Hydroinform. 2015, 17, 347-360. [CrossRef]

29. De Marchis, M.; Milici, B.; Napoli, E. Numerical observations of turbulence structure modification in channel flow over 2D and 3D rough walls. Int. J. Heat Fluid Flow 2015, 56, 108-123. [CrossRef]

30. De Marchis, M.; Milici, B.; Sardina, G.; Napoli, E. Interaction between turbulent structures and particles in roughened channel. Int. J. Multiph. Flow 2016, 78, 117-131. [CrossRef]

31. Vítkovský, J.P.; Bergant, A.; Simpson, A.R.; Lambert, M.F. Systematic evaluation of one-dimensional unsteady friction models in simple pipelines. J. Hydraul. Eng. 2006, 132, 696-708. [CrossRef]

32. De Marchis, M.; Fontanazza, C.M.; Freni, G.; la Loggia, G.; Napoli, E.; Notaro, V. A model of the filling process of an intermittent distribution network. Urban Water J. 2010, 7, 321-333. [CrossRef]

33. Sammartano, V.; Morreale, G.; Sinagra, M.; Tucciarelli, T. Numerical and experimental investigation of a cross-flow water turbine. J. Hydraul. Res. 2016, 54, 321-331. [CrossRef]

34. De Marchis, M. Large eddy simulations of roughened channel flows: Estimation of the energy losses using the slope of the roughness. Comput. Fluids 2016, 140, 148-157. [CrossRef]

35. Marvuglia, A.; Messineo, A. Using recurrent artificial neural networks to forecast household electricity consumption. Energy Procedia 2012, 14, 45-55. [CrossRef]

36. Volpe, R.; Messineo, A.; Millan, M.; Volpe, M.; Kandiyoti, R. Assessment of olive wastes as energy source: Pyrolysis, torrefaction and the key role of $\mathrm{H}$ loss in thermal breakdown. Energy 2015, 82, 119-127. [CrossRef]

37. Volpe, M.; Volpe, R.; Panno, D.; Messineo, A. Upgrade of citrus waste as a biofuel via slow pyrolysis. J. Anal. Appl. Pyrolysis 2015, 115, 66-76. [CrossRef]

38. Alaimo, A.; Esposito, A.; Messineo, A.; Orlando, C.; Tumino, D. 3D CFD analysis of vertical axis wind turbine. Energies 2015, 8, 3013-3033. [CrossRef]

39. De Marchis, M.; Fontanazza, C.; Freni, G.; Notaro, V.; Puleo, V. Experimental evidence of leaks in elastic pipes. Water Resour. Manag. 2016, 30, 2005-2019. [CrossRef]

(C) 2016 by the authors; licensee MDPI, Basel, Switzerland. This article is an open access article distributed under the terms and conditions of the Creative Commons Attribution (CC-BY) license (http://creativecommons.org/licenses/by/4.0/). 